\title{
Lidil
}

Revue de linguistique et de didactique des langues

49 | 2014

L'analyse des données didactiques

Christophe Alix, Dominique Lagorgette et ÈveMarie Rollinat-Levasseur (dir.), Didactique du Français Langue Étrangère par la pratique théâtrale Université de Savoie, Laboratoire LLS, collection «Langages », 2013, $312 \mathrm{p}$.

\section{Sandra Tomc}

\section{OpenEdition}

\section{Journals}

Édition électronique

URL : http://journals.openedition.org/lidil/3523

DOI : $10.4000 /$ lidil.3523

ISSN : 1960-6052

Éditeur

UGA Éditions/Université Grenoble Alpes

Édition imprimée

Date de publication : 25 mai 2014

Pagination : 195-197

ISBN : 978-2-84310-272-1

ISSN : $1146-6480$

\section{Référence électronique}

Sandra Tomc, « Christophe Alix, Dominique Lagorgette et Ève-Marie Rollinat-Levasseur (dir.)

Didactique du Français Langue Étrangère par la pratique théâtrale », Lidil [En ligne], 49 | 2014, mis en ligne le 25 novembre 2015, consulté le 24 septembre 2020. URL : http://journals.openedition.org/lidil/3523 ; DOI : https://doi.org/10.4000/lidil.3523 


\section{Notes de lecture}

Christophe Alix, Dominique Lagorgette et Ève-Marie RollinatLevasseur (dir.), Didactique du Français Langue Étrangère par la pratique théâtrale, Université de Savoie, Laboratoire LLS, coll. "Langages », 2013, 312 p.

Le recueil Didactique du Français Langue Étrangère par la pratique théâtrale réunit des articles de différents champs scientifiques relatifs à l'enseignement du français langue seconde et langue étrangère par la pratique théâtrale. Porté davantage sur la pratique théâtrale que sur l'outil théâtre, ce rapport d'expériences interroge les possibilités de mettre en place des tentatives de jouer, de dire et de faire. Ancrées non pas sur la simple imitation enjouée mais sur une véritable expression explorant la multicanalité de la communication, du canal sonore au canal visuel, les différentes expérimentations se proposent d'amorcer des méthodes pour l'enseignement apprentissage du français dans des cadres hétérogènes (milieu homoglotte, hétéroglotte, accueil de nouveaux/elles migrant-e-s, cadre scolaire et universitaire). Au-delà des situations, une réflexion diachronique sur l'usage pratique du théâtre dans la société est engagée dès l'introduction. Les contributions d'auteur-e-s nous placent d'emblée dans une perspective interdisciplinaire (littérature, didactique, études théâtrales) traitant différents champs théoriques et méthodologiques et diverses expériences d'enseignement. L'objectif est d'instaurer un dialogue s'inscrivant dans une complémentarité, mettant en rapport différentes langues, mais aussi différents contextes d'apprentissage et différent-e-s acteurs/trices. Abordant les problématiques complexes du développement des compétences orales, du plurilinguisme et de l'interculturalité, l'ouvrage incite à trouver des méthodes de recherche transdisciplinaires. Il s'articule en quatre axes.

Le premier volet s'organise autour de la multiplicité des questions théoriques induite par le théâtre dans l'enseignement apprentissage du français et des explorations didactiques des pratiques théâtrales. Les articles ont pour but de montrer ce que recouvre l'activité mimétique dans des contextes éducatifs historiquement attestés, participant à l'émergence de réflexions sur le statut des représentations de la langue d'apprentissage. C'est à partir de ce cadre que sont exposés des fondements 
sur l'identité et l'altérité, aux prises avec une nouvelle langue-culture. L'exploration didactique des pratiques théâtrales soulève des questions susceptibles d'agir sur «l'authenticité» de l'individu, questionnée au travers de ses rapports à la parole, à la voix, au corps, aux languescultures et aux différentes œuvres. De la même façon, les exercices de pré-expressivité associés à des notions d'anthropologie théâtrale apportent une contribution importante aux pratiques orales et à l'accès au sens.

C'est autour du paradigme de la pratique théâtrale que se confrontent, dans la deuxième partie, des expériences et des analyses des différentes méthodologies à l'œuvre dans des contextes spécifiques. Cette perspective traverse tous les textes de ce volet, dans lesquels l'objectif est de montrer que la protection qu'offre l'espace scénique à l'apprenant-e, doit lui permettre d'exploiter ses capacités de création et d'expression et lui fournir des apports en termes d'attitudes envers la langue à apprendre, envers l'apprentissage individuel et collectif. Se côtoient tout au long des situations, des activités pensées dans une perspective de contextualisation. La méthodologie socioconstructiviste place l'interaction et la réflexion au centre du processus, permettant à l'apprenant-e acteur/ trice l'instauration d'un climat de confiance dans la communauté d'apprentissage. À travers des approches plurielles telles que l'utilisation de textes littéraires et de contes, des constructions de cours expérimentaux sont successivement présentés dans une perspective sociodidactique. Si on s'appuie sur leur appropriation, leur découpage pour mieux les reconstruire, leur déclinaison sous des formes variées, on voit qu'ils sont autant d'occasion d'ouverture à l'interculturel, à la rencontre de la langue de l'autre, autant de pistes de travail qui placent l'apprenant-e en situation d'acteur/trice au cours du travail comme sur la scène.

Les chapitres du troisième volet prennent pour cible l'articulation entre les pratiques théâtrales et les pratiques langagières. La pratique théâtrale offre aux apprenant-e-s un véritable espace de mise en je. C'est ce que se proposent de montrer les auteur-e-s en présentant des contextes reflétant la prise en compte d'inférences linguistiques dans des sketches, des exercices d'amplification pour appréhender la phonétique, la méthode verbo-tonale «corrective» permettant de considérer la variation. La perspective identitaire traverse toutes ces mises en œuvre, en privilégiant la façon dont les sujets attribuent du sens à leurs actions.

Dans la dernière partie de l'ouvrage, des pistes d'exploration d'écritures et de pratiques théâtrales contemporaines sont proposées. On y interroge leurs intérêts ludiques et poétiques, leurs effets de décentre- 
ment et leurs possibilités pour affiner les interactions. Au travers de la pratique, les apprenant-e-s disposent d'une palette d'expressivité suffisamment large pour tisser des ponts entre eux et la société culturelle qui les entoure. Ces nouvelles pistes de didactisation participent à une meilleure compréhension des textes et de la langue, et offrent la perspective d'établir des passerelles vers d'autres langages. De la même façon, l'articulation entre ici et là-bas prend toute son ampleur dans l'utilisation des pratiques théâtrales chez les migrant-e-s.

Sandra Tome CELEC et Université Jean Monnet - Saint-Étienne

Langage et Société, $\mathrm{n}^{\circ}$ 145, "Enjeux sociaux des mouvements de revitalisation linguistique ", dirigé par James Costa, Éditions de la Maison des sciences de l'homme, 2013

Dans ce numéro, James Costa et les auteur-e-s qu'il a réuni-e-s s'attaquent courageusement à la question des langues minorées, victimes trop souvent de discours répétitifs et donc contre-productifs. En effet, comme l'écrit ce chercheur, «bien souvent, le débat autour de ces problématiques se résume à des prises de position passionnées "pour" ou "contre" ces langues». Les textes rassemblés veulent renouveler le regard et donc le discours sur ces langues, appelées aussi «régionales» ou «minoritaires», sans qu'aucune appellation soit jamais satisfaisante.

Et ils renouvellent ce discours à plusieurs titres :

- d'abord en portant sur les mouvements qui les ont fait connaitre un regard résolument sociolinguistique qui «s'interrog(e) sur le sens social des demandes qu'expriment les militants qui les défendent»;

- ensuite en choisissant d'observer des mouvements de «revitalisation» plutôt que de développer, comme à l'ordinaire, des discours de «mise en danger» et de «sauvetage urgent» de ces langues;

- enfin en préférant approfondir quelques cas peu connus (vision historique sur les «renaissantismes en Provence», textes sur la «promotion institutionnelle de l'irlandais », et sur une langue du Nicaragua). 